\title{
Spatial perception and control
}

\author{
J. SCOTT JORDAN \\ Illinois State University, Normal, Illinois \\ and \\ GÜNTHER KNOBLICH \\ Max Planck Institute for Psychological Research, Munich, Germany
}

\begin{abstract}
We investigated whether the perceived vanishing point of a moving stimulus becomes more accurate as one's degree of control over the stimulus increases. Either alone or as a member of a pair, participants controlled the progression of a dot stimulus back and forth across a computer monitor. They did so via right and left buttonpresses that incremented the dot's velocity rightward and leftward, respectively. The participants in the individual condition had control of both buttons. Those in the group condition had control of only one. As the participants slowed the dot to change its direction of travel, it unexpectedly disappeared. Localizations of the vanishing point became more accurate as the participants' control over the dot increased. The data bridge a gap between accounts of localization error that rely solely on stimulus and cognitive factors, and accounts derived from research on action and spatial perception, which tend to rely on action-planning factors.
\end{abstract}

When participants are asked to indicate the vanishing point of a moving or apparently moving stimulus, the perceived vanishing point tends to be displaced beyond the actual vanishing point (Finke, Freyd, \& Shyi, 1986; Freyd \& Finke, 1984, 1985; Hubbard, 1995b; Hubbard \& Motes, 2002). In addition, the magnitude of the displacement varies as a function of both stimulus properties and cognitive processes. Examples of the former include increases in displacement due to increased velocity (Hubbard \& Bharucha, 1988), decreases due to increased surface contact (i.e., friction; Hubbard, 1995a, 1998), and decreases due to upward versus downward stimulus travel (i.e., gravity; Hubbard, 1990, 1997). Examples of the latter include variations in (1) displacement direction (i.e., behind vs. beyond) due to expectancy of a stimulus/barrier collision (Hubbard, 1994), (2) displacement magnitude due to stimulus identity (i.e., the stimulus is identified as a rocket vs. a church steeple; Reed \& Vinson, 1996; Vinson \& Reed, 2002), and (3) displacement direction due to launching effects (Hubbard, Blessum, \& Ruppel, 2001).

Recent experiments have indicated that in addition to cognitive and stimulus factors, action-related factors influence localization error as well. Kerzel, Jordan, and Müsseler (2001), for example, found that if participants are allowed to track the moving stimulus via eye movements (i.e., the pursuit condition), the usual displacement

We thank Ian Thornton, Chris Frith, Iring Koch, and two anonymous reviewers for their helpful comments. We also thank Irmgard Hagen, Lucia Kypcke, and Patrick Bach for their help in collecting the data and Marc Grosjean for his help with analyzing the data. Correspondence concerning this article should be addressed to J. S. Jordan, Department of Psychology, Illinois State University, Campus Box 4620, Normal, IL 61790-4620(e-mail: jsjorda@ilstu.edu). beyond the vanishing point obtains. If, however, they are asked to fixate a nonmoving stimulus during the presentation of the moving stimulus (i.e., the fixation condition), localization scores are either veridical or behind the vanishing point. Research by Jordan, Stork, Knuf, Kerzel, and Müsseler (2002) indicated that these actionrelated differences are due to the effect one plans in relation to the moving stimulus, not to the actions used to attain the planned effect. Specifically, they replicated the fixation condition of Kerzel et al.'s study, save for the following: (1) The stimulus trajectory was circular (i.e., around the fixation point), as opposed to linear, and (2) the disappearance of the stimulus was produced by the participants via a buttonpress. If the participants were instructed to press the button in order to make the moving stimulus vanish (i.e., the intention condition), there was no localization error, regardless of stimulus velocity. If, however, they were instructed to press the button in response to the onset of the moving stimulus (i.e., the cue condition), localization scores were behind the actual vanishing point in the direction of the stimulus's initial position, and their size did not vary with velocity. These data are, in general, consistent with the view that action planning modulates localization error, because in both conditions localization scores were attracted to the stimulus location specified in the action plan (i.e., the final and initial positions in the intention and the cue conditions, respectively), despite the fact that the participants produced the same actions in both conditions (i.e., fixated centrally and produced a buttonpress). To ensure that these differences were due to action planning, and not to trajectory length (i.e., the stimulus sketched out a shorter trajectory in the cue condition, since the participants pressed the button as soon as the stimulus ap- 
peared), there was a control condition in which trajectory length was comparable to that in the cue condition, yet the disappearance of the stimulus was produced by the computer (i.e., the induction condition). In this condition, localization scores seemed to be influenced more by stimulus velocity than by action planning, for the scores were slightly beyond the vanishing point at slower velocities, yet were significantly behind the vanishing point at faster velocities.

Collectively, these data are consistent with the notion that action planning modulates localization error. In addition, they indicate that the modulation derives from the type and degree of anticipation that participants had to reflect in the action plans they generated in relation to the moving stimulus. In the pursuit condition of Kerzel et al.'s (2001) study, participants had to control eye-target relationships. Thus, their action plans had to continuously specify future eye-target coordinations that took into account anticipated target locations. In short, the planned effect referred to future events. This anticipatory aspect of the planned effect may have biased the localization scores beyond the vanishing point by an amount directly related to stimulus velocity or, from a planning perspective, the degree of anticipation that had to be reflected in the planned action. In the fixation and induction conditions in Kerzel et al.'s and Jordan et al.'s (2002) studies, respectively, participants controlled their relationship to the fixation point, not to the moving stimulus. Thus, their action plans did not have to integrate anticipated future locations of the moving stimulus, and localizations of the vanishing point were either close to the vanishing point or behind it and were dominated by stimulus factors. Finally, in the intention condition in Jordan et al.'s study, participants controlled the vanishing point (i.e., they specified it and then produced it via their actions), and there was no localization error, regardless of stimulus velocity.

Taken as a whole, these data imply a rather straightforward relationship between control, anticipation, and localization error. Specifically, the better control one has over a moving stimulus, the smaller the localization error. To test this implication, we devised a task in which participants controlled stimulus motion endogenously, as opposed to the computer doing so exogenously. Specifically, the participants were asked to move a dot back and forth on a computer monitor as quickly as possible (see Figure 1). They did so by pressing buttons (i.e., a left and a right one) that either accelerated or decelerated the dot, depending on its direction of travel. If the dot was traveling to the right, right buttonpresses produced acceleration, and left buttonpresses produced deceleration. If the dot was traveling to the left, the opposite relationships obtained. In order to change the dot's directionfrom rightward to leftward, for example-the participants first had to decelerate its rightward motion (i.e., they had to "brake" the dot) and then accelerate it to the left. At an unpredictable moment during the braking

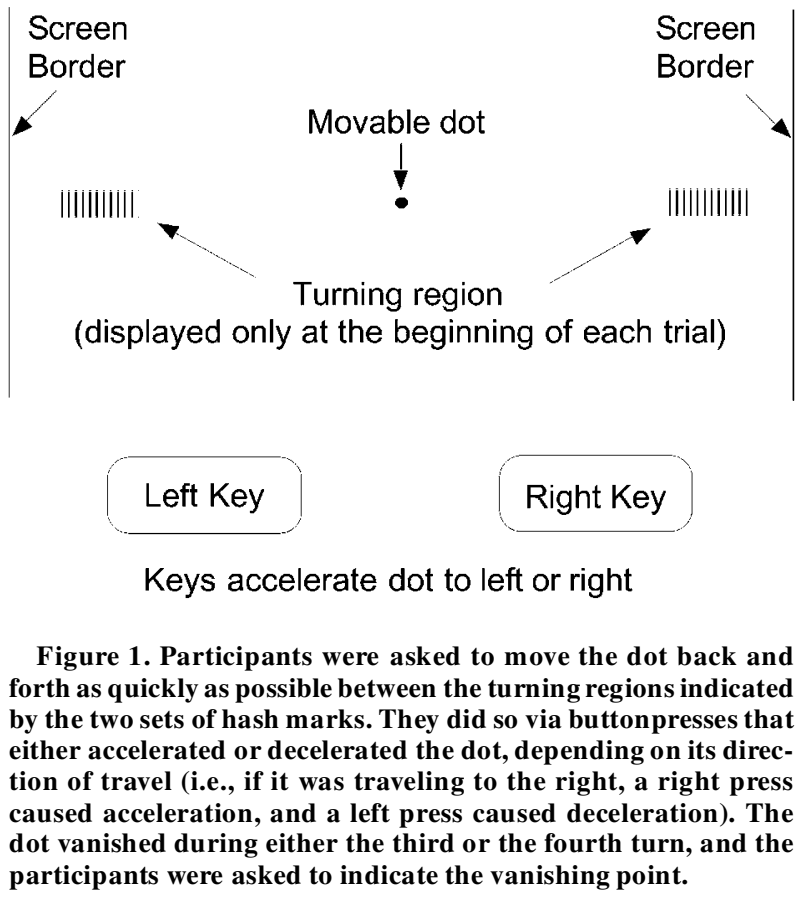

phase, the dot vanished, and the participants were asked to indicate its vanishing point.

Effective endogenous control during a braking phase requires action plans that take into account future states. That is, as a participant presses the buttons to control the deceleration of the dot, the plans to do so must entail anticipation regarding the degree of deceleration each buttonpress actually produces. Otherwise, the participant may produce an inappropriate sequence of presses, lose control of the dot's deceleration, and allow it to move off the screen. If the localization error decreases with better control, an increase in the degree of deceleration the participant can anticipate with each buttonpress should, as long as the deceleration is not too great (i.e., the gain is not too high), make the task easier (i.e., increase the quality of control) and result in smaller localization errors (i.e., anticipating more deceleration per buttonpress leads to less localization error).

To be sure, control in this context is being defined as a participant's attempt to decelerate the dot via buttonpresses, and quality of control is being defined in terms of the ease with which a participant is able to affect such control. Given these definitions, we manipulated quality of control in two ways. First, we varied the amount of deceleration/acceleration produced by a single buttonpress. The logic behind this manipulation was that increasing the impact of the buttonpresses would increase the quality of control because braking would be easier with high-versus low-impact presses (i.e., the former would require fewer presses). Given that controlling deceleration with high-impact presses requires more anticipated deceleration per buttonpress, localization error 
should be smaller in the high-impact condition (i.e., greater anticipated deceleration gives rise to less error).

The second manipulation varied the participants' quality of control in a somewhat different manner. Specifically, we had the participants do the task either alone or as a member of a pair. In the individual condition, the participants had access to both buttons, whereas in the group condition each had access to only one. The logic behind this manipulation was that having access to both buttons, as opposed to only one, would increase the participants' quality of control, because it would afford direct access to knowledge regarding when and if either button was to be pressed. This in turn would directly influence the type of anticipation the participants could realistically generate as they controlled the dot's deceleration. When the participants in the group condition planned to decelerate the dot, their lack of knowledge regarding when and if the other button was to be pressed would prevent them from anticipating as much deceleration per buttonpress as would the participants in the individual condition. Given these differences in anticipated deceleration, the localization error should be smaller in the individual condition (i.e., greater anticipated deceleration gives rise to less error). Finally, we had the participants localize the vanishing point in the group condition regardless of who was decelerating the dot at the moment it vanished. If the dot vanished while the "other" was braking, the participants' quality of control would be at a minimum, because he/she has been rendered an observer yet still has to remain engaged in the task in order to know when to take control over the dot. While functioning in this capacity, the participants would not be able to anticipate as much deceleration as would the member of the pair actually controlling the dot's deceleration. These differences in anticipated deceleration should result in less error when the "self" versus the "other" was responsible for the dot's deceleration (i.e., greater anticipated deceleration gives rise to less error). In summary, as the quality of the participants' control over the dot's deceleration increases (i.e., self vs. other control, individual vs. group control, and high vs. low impact), the degree of anticipated deceleration per buttonpress should increase, and the size of the localization error should, as a result, decrease.

\section{METHOD}

\section{Participants}

A total of 41 participants took part in the experiment. They were recruited by advertising at the University of Munich and in local papers and were paid for their participation. There were 17 in the individual condition. Their ages ranged from 17 to 31 years, and 6 of them were male. There were 24 in the group condition. Their ages ranged from 19 to 30 years, and 8 of them were male. All the participants had normal or corrected-to-normal vision.

\section{Materials and Procedure}

Upon entering the lab, the participants were informed of the nature of the task. Afterward, they were seated in front of a computer monitor at a distance of $60 \mathrm{~cm}$. Those in the individual condition were given a control panel consisting of a left and a right key. The participants in the group condition were given a panel with only one of the two keys. A laboratory assistant controlled the other key. Key assignment in the group condition was counterbalanced across participants. In addition, the assistant and the participant were divided by a partition, and each had a separate computer monitor. All events were presented simultaneously on both monitors. During training, the assistant was allowed to talk to the participant in order to facilitate learning of the task. During the main experiment, however, the assistant was silent.

The task was to move a dot between the two screen borders as fast as possible. Keypresses either accelerated or decelerated the dot, depending on its direction of travel. When it was traveling to the left, left presses produced acceleration, and right presses produced deceleration. If the dot was traveling to the right, the opposite relationships obtained. To ensure that the stimulus display was similar across different participants, the task required that the direction of the dot be turned within a certain area near the edge of the monitor (see Figure 1). In addition, the participants had to ensure that the dot reached a minimal peak velocity of $9.10 \mathrm{deg} / \mathrm{sec}$ prior to the onset of braking.

Simultaneous keypresses negated one another. Given the task demands, however (i.e., attain the minimum required velocity, yet ensure that the dot changes direction within the turning zone), the participants in the group condition had to learn to cooperate and not interfere with the other's attempts to accelerate or decelerate the dot. Thus, by the onset of the experimental trials, the participants had learned the pattern of presses necessary for successful completion of a trial and did not produce conflicting responses.

The course of each trial was as follows. A solid rectangle (size, $0.4^{\circ}$ of visual angle) was displayed in the middle of the screen. In addition, the valid turning areas on both sides of the screen were indicated by hatched rectangles (see Figure 1). Each turning area subtended a visual angle of $3.1^{\circ}$. The distance of the near border of each area from the screen border was $1.9^{\circ}$. The turning areas were removed from the screen border to control for landmark effects on perceived vanishing point (Hubbard \& Ruppel, 1999, 2000). At the beginning of each trial, the participant clicked the rectangle. Immediately thereafter, the rectangle was replaced by a circular solid dot, and the turning area markers vanished. As soon as a button was pressed, the dot started moving to the right. The participants were allowed to move their eyes in order to track the dot. Each keypress accelerated or decelerated the dot by $1.1 \mathrm{deg} / \mathrm{sec}^{2}$ in the low-impact condition and by $1.7 \mathrm{deg} / \mathrm{sec}^{2}$ in the high-impact condition. Although these constituted stepwise increments/decrements of the dot's velocity, they were small enough to ensure that the changes in the dot's velocity were always smooth and gradual. The participants received an error message if (1) they tried to turn the dot outside of the valid turning areas or (2) they did not reach the minimal peak velocity required before each turn. Trials including such errors were repeated.

The dot disappeared before either the third or the fourth turn. The exact turn varied randomly across trials. This ensured that the participants actually had control over the dot at the moment it vanished. In addition, it ensured that the dot would vanish an equal number of times on both sides of the monitor (i.e., right and left). In order to control for velocity effects on the perceived vanishing point (Hubbard, 1995b; Hubbard \& Bharucha, 1988), the dot disappeared immediately following the first buttonpress made after the dot had been decelerated to a velocity of $6.9 \mathrm{deg} / \mathrm{sec}$. This was the case for both levels of impact. After a 500-msec delay, a crosshair appeared in the middle of the screen. The participants moved the crosshair, via a computer mouse, in the same horizontal plane as the dot and indicated the dot's perceived vanishing point by pressing a button on the mouse. The participants in the group condition did so both when the dot vanished while under their control (i.e., the group-act condition) and when it vanished while under the assistant's control (i.e., the group-observe condition). 
The participants underwent 20 training trials, during which they were not asked to indicate the vanishing point. Following training, the participants underwent 10 additional trials in which they did indicate the vanishing point. Then the main experiment entailed three 40-trial blocks. The order of trials within a block was randomized.

\section{RESULTS}

The distance between the actual and the indicated vanishing points was measured in terms of visual angle and was used as the dependent variable. Positive and negative values indicate localization scores beyond and behind the actual vanishing point, respectively. Figure 2 illustrates the results. Mean localization error was greater than zero (i.e., beyond the vanishing point) in all conditions. In the individual condition, it was $0.44^{\circ}$ of visual angle $(s=0.030)$ at low impact and $0.37^{\circ}(s=0.030)$ at high impact. In the group-act condition, it was $0.52^{\circ}(s=$ $0.027)$ and $0.38^{\circ}(s=0.027)$, at low and high impact, respectively. Finally, in the group-observe condition, mean displacement was $0.59^{\circ}(s=0.027)$ and $0.50^{\circ}(s=$ 0.027), at low and high impact, respectively.

To assess whether or not localization error varied with the quality of control, we conducted two analyses of variance (ANOVAs). Whereas the first was a $2 \times 2$ mixed ANOVA with condition (individual vs. group-act, between subjects) and impact (low vs. high, within subjects) as factors, the second was a $2 \times 2$ repeated measures ANOVA with condition (group-observe vs. group-act) and impact of buttonpress (low vs. high, within subjects) as factors. We conducted separate $2 \times 2$ ANOVAs, versus one $3 \times 2$ ANOVA, because the individual versus

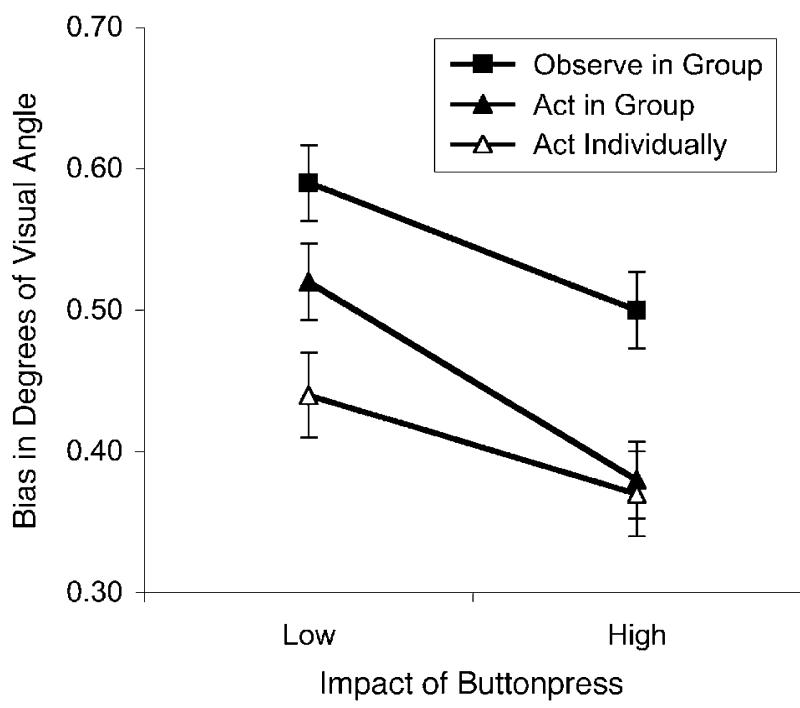

Figure 2. Localization bias in the individual, group-act, and group-observe conditions. Given that the comparison across impact conditions is within subjects, error bars were determined according to Loftus and Masson (1994). The error bars for the group (i.e., group-act and group-observe) and the individual data reflect the error terms of the within- and between-subjects analyses of variance, respectively. group-act comparison was between subjects, whereas the group-act versus group-observe comparison was within subjects.

In the individual versus group-act analysis, there was a main effect for impact $[F(1,39)=54.3, p<.001]$, no main effect of condition, and a significant interaction between the two $[F(1,39)=7.0, p<.05]$. Specifically, the error was significantly smaller in the high-impact condition. It was also smaller in the individual, versus the group-act, condition, but only at low impact. At high impact, there was no significant difference between the individual and the group-act conditions.

In the group-act versus group-observe analysis, there were significant main effects for both impact $[F(1,23)=$ $66.4, p<.001]$ and condition $[F(1,23)=5.2, p<.05]$, but no significant interaction. Specifically, errors were smaller in both the high-impact condition and the groupact condition.

\section{DISCUSSION}

The purpose of the present study was to determine whether or not the perceived vanishing point of a moving stimulus becomes more accurate as one's control over the moving stimulus increases. The present data seem to support this assertion. Error was significantly smaller in the high-impact condition in both analyses (i.e., individual/group-act and group-act/group-observe) and was at its lowest when individuals controlled the dot themselves. According to an action-planning account, higher impact presses made the task easier and allowed the participants to reflect greater anticipated deceleration in their action plans, which, in turn, reduced the localization error. Furthermore, being engaged in the control task, yet being in a position of withholding one's only available response (i.e., the group-observe condition), reduced the participants' quality of control over the task to such an extent that the amount of anticipated deceleration was at its minimum, thus giving rise to the greatest error.

As for the interaction in the individual versus groupact analysis, the data do, with some qualification, support the quality-of-control account. What was predicted was a main effect, with the error being smallest in the individual condition. This turned out to be true at low impact only. From an action-planning perspective, this pattern suggests that the amount of deceleration produced by high-impact buttonpresses was so robust, within the context of the present task demands, that the group-act participants were able to anticipate roughly the same degree of deceleration per buttonpress as those in the individual condition, even though they had access to only one button. Such an account is, of course, speculative. The overall pattern, however, is rather clear. Localization error generally decreases as one's control over a stimulus increases.

To be sure, one might argue that an action-planning account is unnecessary. For example, one might argue 
that the impact effect occurred because the different rates of deceleration in the two conditions gave rise to different action-independent cognitive expectancies about the degree of postdisappearance stimulus motion. Such an account, however, cannot explain the groupact/group-observe difference. Stimulus parameters (i.e., minimum required velocity and vanishing velocity) were the same in both conditions. Thus, the only difference between the two was the quality of control the participants had over the dot at the moment it vanished. One might counter by arguing that the assistant may have used a different strategy than the participants, thus giving rise to different stimulus patterns in the two group conditions. We measured performance in a very similar paradigm, however (Knoblich \& Jordan, 2003), and found that successful cooperation requires patterns of buttonpresses that are alike, both across different groups and in comparison with individuals doing the task alone. Thus, the necessary pattern of presses was basically fixed by task demands and is not likely to have caused differences in the display that can account for the present data.

In addition, the present data fall within an actiondependent pattern of localization error that extends across multiple experiments, including those involving exogenous stimulus control. In the cue and intention conditions of Jordan et al.'s (2002) study and in all the conditions of the present study, the stimulus vanished as a consequence of the participants' buttonpress, yet the error progressed from negative, to zero, to positive as the temporal frame reflected in the action plan progressed from past (the initial position in Jordan et al.'s cue condition) to pending present (the actual vanishing point in their intention condition) to the more distant future (decelerating the dot in the present experiment). This pattern also extends into the pursuit condition of the Kertzel et al. (2001) study in which participants had no control over the stimulus yet tracked it via eye movements. In this condition, which constituted a replication of the typical exogenous case, localization errors were beyond the vanishing point, were much larger than those in the present study, and increased as the requisite tracking velocity increased (i.e., stimulus velocity increased). Given this pattern, the present data seem more coherently accounted for via action-planning mechanisms versus action-independent factors, such as stimulus properties and/or cognitive mechanisms.

As to the nature of the action-planning mechanisms underlying the modulation, research on attention (Bachmann, 1999; Klein, 1988; Posner, 1980; Posner \& Cohen, 1984; Schneider \& Deubel, 2002; Wolff, 1999) indicates that roughly 50-100 msec after the presentation of a saccadic target, the threshold for the detection of events at the target's position is reduced. Such presaccadic shifts in detectability thresholds constitute shifts in spatial perception that are related to action planning and may reflect the functioning of a mechanism that allows anticipation to be reflected within action plans. Another possibility is the theory of event coding (TEC; Hommel, Müsseler, Aschers- leben, \& Prinz, 2001), which asserts that (1) actions are planned in terms of the distal effects they are to produce and (2) perception and action planning share common neural resources. According to TEC, planning to decelerate the dot (i.e., a distal effect) would simultaneously alter the perceptual mappings of the dot in a planningrelative fashion. As the planned effect required more and more anticipated deceleration, the perceptual mappings of the stimulus would be less and less biased, thus giving rise to more accurate localization scores.

Deciding between these two accounts proves difficult. TEC posits a functional coupling between action planning and perceptual mappings, it accounts for all actionrelative shifts in localization error, and it does so rather parsimoniously. An attentional account could perhaps do the same, despite the fact that it posits no functional coupling between action planning and perceptual mapping and, as a consequence, would require additional mechanisms to fully account for action-related localization error. Regardless, however, it is not at all clear that the two approaches are empirically distinguishable, for there are those who argue (Rizzollatti, Riggio, Dascola, \& Umiltà, 1987; Wolff, 1999) that preaction shifts in attention constitute a necessary component of action planning. Deciding between the two, therefore, is not, at present, a clear-cut issue.

In closing, the present data provide rather strong support for the assertion that localization error is modulated by action planning and that the degree of localization error decreases as one's control over a moving stimulus increases. An immediate benefit of adding action planning to the list of factors underlying localization error is that it bridges a gap between previous research on localization error, which relied primarily on stimulus and cognitive accounts, and research on spatial perception, which relies heavily on action-related mechanisms, such as attention and/or common coding (i.e., TEC). Given its ability to bridge this gap, action planning seems to merit a special place among an increasing number of factors that have been found to influence localization error (Nagai \& Saiki, 2001; Senior et al., 1999; Stork \& Müsseler, 2001; Thornton, 2002).

\section{REFERENCES}

BACHMANN, T. (1999). Twelve spatiotemporal phenomena and one explanation. In G. Aschersleben, J. Müsseler, \& T. Bachmann (Eds.), Cognitive contributions to the perception of spatial and temporal events (pp. 173-206). Amsterdam: Elsevier, North-Holland.

Finke, R. A., FreYd, J. J., \& ShYI, G. C. W. (1986). Implied velocity and acceleration induce transformations of visual memory. Journal of Experimental Psychology: General, 115, 175-188.

FREYD, J. J., \& FINKE, R. A. (1984). Representational momentum. Journal of Experimental Psychology: Learning, Memory, \& Cognition, 10, 126-132.

FREYD, J. J., \& FINKE, R. A. (1985). A velocity effect for representational momentum. Bulletin of the Psychonomic Society, 23, 443-446.

Hommel, B., Müsseler, J., Aschersleben, G., \& Prinz, W. (2001). The theory of event coding (TEC): A framework for perception and action planning. Behavioral \& Brain Sciences, 24, 849-937.

HubBARD, T. L. (1990). Cognitive representation of linear motion: Pos- 
sible direction and gravity effects in judged displacement. Memory \& Cognition, 18, 299-309.

Hubbard, T. L. (1994). Judged displacement: A modular process? American Journal of Psychology, 107, 359-373.

HubBARD, T. L. (1995a). Cognitive representation of motion: Evidence for friction and gravity analogues. Journal of Experimental Psychology: Learning, Memory, \& Cognition, 21, 241-254.

HuBBARD, T. L. (1995b). Environmental invariants in the representation of motion: Implied dynamics and representational momentum, gravity, friction, and centripetal force. Psychonomic Bulletin \& Review, 2, 322-338.

HubBARD, T. L. (1997). Target size and displacement along the axis of implied gravitational attraction: Effects of implied weight and evidence of representational gravity. Journal of Experimental Psychology: Learning, Memory, \& Cognition, 23, 1484-1493.

HubBARD, T. L. (1998). Some effects of representational friction, target size, and memory averaging on memory for vertically moving targets. Canadian Journal of Experimental Psychology, 52, 44-49.

Hubbard, T. L., \& BharuCha, J. (1988). Judged displacement in apparent vertical and horizontal motion. Perception \& Psychophysics, 44, 211-221.

Hubbard, T. L., Blessum, J., \& RupPel, S. (2001). Representational momentum and Michotte's "launching effect" paradigm. Journal of Experimental Psychology: Learning, Memory, \& Cognition, 27, 294301.

Hubbard, T. L., \& Motes, M. (2002). Does representational momentum reflect a distortion of the length or the endpoint of a trajectory? Cognition, 82, B89-B99.

HubBARD, T. L., \& RuPPEL, S. (1999). Representational momentum and the landmark attraction effect. Canadian Journal of Experimental Psychology, 53, 242-256.

Hubbard, T. L., \& RuPPel, S. (2000). Spatial memory averaging, the landmark attraction effect, and representational gravity. Psychological Research, 64, 41-55.

Jordan, J. S., Stork, S., KNuf, L., KerZel, D., \& MüsSeler, J. (2002). Action planning affects spatial localization. In W. Prinz \& B. Hommel (Eds.), Attention and performance XIX: Common mechanisms in perception and action (pp. 158-176). Oxford: Oxford University Press.

KERZEL, D., JoRDAN, J. S., \& MüSSE LER, J. (2001). The role of perceptual anticipation in the localization of the final position of a moving target. Journal of Experimental Psychology: Human Perception \& Performance, 27, 829-840.

KLEIN, R. (1988). Inhibitory tagging system facilitates visual search. Nature, 334, 430-431.
KNOBLICH, G., \& JORDAN, J. S. (2003). Action coordination in groups and individuals: Learning anticipatory control. Journal of Experimental Psychology: Learning, Memory, \& Cognition, 29, 1006-1016.

LofTus, G. R., \& MAsson, M. E. J. (1994). Using confidence intervals in within-subject designs. Psychonomic Bulletin \& Review, 1, 476-490.

Nagai, M., \& SAIKI, J. (2001, November). Direction of forward memory displacement is different from perceived motion direction. Paper presented at the 42nd Annual Meeting of the Psychonomic Society, Orlando, FL.

Posner, M. I. (1980). Orienting of attention. Quarterly Journal of Experimental Psychology, 32, 3-25.

Posner, M. I., \& COHEN, Y. (1984). Components of visual orienting. In H. Bouma \& D. G. Bouwhuis (Eds.), Attention and Performance $X$ : Control of language processes (pp. 531-556). Hillsdale, NJ: Erlbaum.

REED, C., \& VINSON, N. (1996). Conceptual effects on representational momentum. Journal of Experimental Psychology: Human Perception \& Performance, 22, 839-850.

Rizzolatti, G., Riggio, L., Dascola, I., \& Umiltà, C. (1987). Reorienting attention across the horizontal and vertical meridians: Evidence in favor of a premotor theory of attention. Neuropsychologia, 25, 31-40.

SCHNeIder, W., \& Deubel, H. (2002). Selection-for-perception and selection-for-spatial-motor-action are coupled by visual attention: A review of recent findings and new evidence from stimulus-driven saccade control. In W. Prinz \& B. Hommel (Eds.), Common mechanisms in perception and action: Attention and performance XIX (pp. 609-627). Oxford: Oxford University Press.

Senior, C., Barnes, J., Giampietro, V., Simmons, A., Bullmore, E. T., BRAMMER, M., \& DAVID, A. S. (1999). The functional neuroanatomy of implicit-motion perception or "representational momentum." Current Biology, 10, 16-22.

STORK, S., \& MÜSSE LER, J. (2001, November). Saccadic eye movements and mislocalizations of briefly presented stimuli. Paper presented at the 42nd Annual Meeting of the Psychonomic Society, Orlando, FL.

Thornton, I. M. (2002). The onset repulsion effect. Spatial Vision, 15, 219-243.

Vinson, N., \& REED, C. (2002). Sources of object-specific effects in representational momentum. Visual Cognition, 9, 41-65.

WoLFF, P. (1999). Space perception and intended action. In G. Aschersleben, J. Müsseler, \& T. Bachmann (Eds.), Cognitive contributions to the perception of spatial and temporal events (pp. 43-63). Amsterdam: Elsevier, North-Holland.

(Manuscript received February 11, 2002; revision accepted for publication October 25, 2002.) 